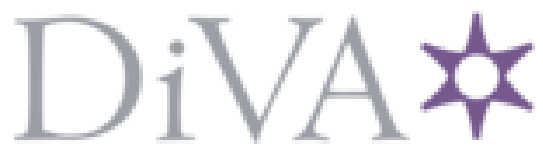

http://www.diva-portal.org

This is the published version of a paper published in Journal of Physical Chemistry Letters.

Citation for the original published paper (version of record):

Skrypnychuk, V., Boulanger, N., Nordenström, A., Talyzin, A V. (2020)

Aqueous Activated Graphene Dispersions for Deposition of High-Surface Area

Supercapacitor Electrodes

Journal of Physical Chemistry Letters, 11(8): 3032-3038

https://doi.org/10.1021/acs.jpclett.oco0272

Access to the published version may require subscription.

N.B. When citing this work, cite the original published paper.

Permanent link to this version:

http://urn.kb.se/resolve?urn=urn:nbn:se:umu:diva-169134 


\title{
Aqueous Activated Graphene Dispersions for Deposition of High- Surface Area Supercapacitor Electrodes
}

\author{
Vasyl Skrypnychuk, Nicolas Boulanger, Andreas Nordenström, and Alexandr Talyzin*
}

Cite This: J. Phys. Chem. Lett. 2020, 11, 3032-3038

Read Online

ABSTRACT: High-surface area activated graphene has a threedimensional porous structure that makes it difficult to prepare dispersions. Here we report a general approach that allows the preparatioon of stable water-based dispersions/inks at concentrations of $\lesssim 20 \mathrm{mg} / \mathrm{mL}$ based on activated graphene using environmentally friendly formulations. Simple drying of the dispersion on the substrate allows the preparation of electrodes that maintain the high specific surface area of the precursor material $\left(\sim 1700 \mathrm{~m}^{2} / \mathrm{g}\right)$. The electrodes are flexible because of the structure that consists of micrometer-sized activated graphene grains interconnected by carbon nanotubes (CNTs). The

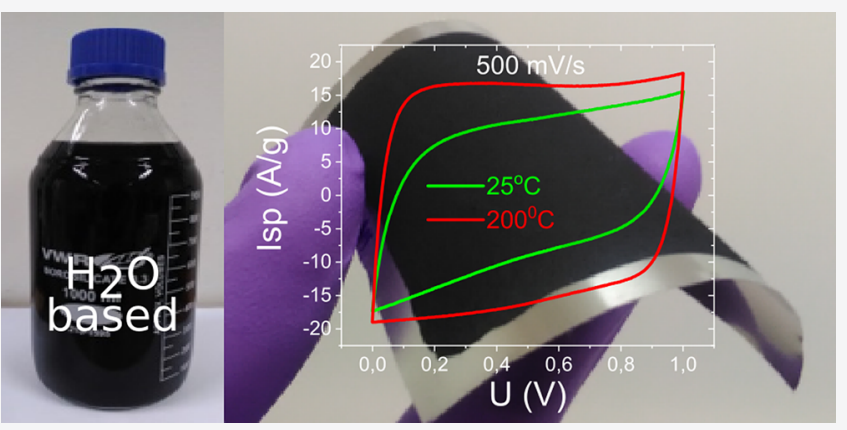
electrodes prepared using activated graphene demonstrate performance superior to that of reduced graphene oxide in supercapacitors with $\mathrm{KOH}$ and $\mathrm{TEA} \mathrm{BF}_{4} /$ acetonitrile electrolytes providing specific capacitance values of 180 and $137 \mathrm{~F} / \mathrm{g}$, respectively, at a specific current of $1 \mathrm{~A} / \mathrm{g}$. The high surface area of activated graphene in combination with the good conductivity of CNTs allows an energy density of $35.6 \mathrm{Wh} / \mathrm{kg}$ and a power density of $42.2 \mathrm{~kW} / \mathrm{kg}$ to be achieved. The activated graphene dispersions were prepared in liter amounts and are compatible with most industrial deposition methods.

S upercapacitors are a very promising solution for energy storage. ${ }^{1}$ The key advantages of supercapacitors compared to batteries are the superior power density and more stable performance with a significantly higher number of chargedischarge cycles. ${ }^{2}$ Commercial supercapacitors are typically produced using porous carbon materials, e.g., activated carbons. Many other kinds of nanostructured carbon materials and composites have been tested on the laboratory scale, aiming to improve the energy density of supercapacitors, 3,4 which remains lower than those of batteries.

Graphene is a promising material for the preparation of supercapacitor electrodes ${ }^{5}$ because of the combination of a high theoretical specific surface area (SSA $\sim 2630 \mathrm{~m}^{2} / \mathrm{g}$ ) and good conductivity. ${ }^{6}$ However, bulk graphene-related materials (GRMs), e.g., most commonly reduced graphene oxide (rGO), demonstrate much lower BET SSA values of 300$800 \mathrm{~m}^{2} / \mathrm{g}$. ${ }^{7,8}$ Moreover, the SSA values are most often reported only for precursor materials rather than for ready-touse electrodes.

The precursor graphene needs to be assembled into a threedimensional (3D) structure to make supercapacitor electrodes, which requires the use of different types of spacers to prevent restacking into graphitic structure, binders, and other added components. A significant drop in SSA occurs in most of the procedures required for the preparation of electrodes, depending on how individual graphene sheets are reas- sembled. ${ }^{9,10}$ The assembly procedure is also decisive for the formation of pores in the electrode structure.

In contrast, "activated graphene" (a-rGO) prepared using $\mathrm{KOH}$ activation of rGO has a rigid 3D structure and welldefined pore size. The unique combination of a high surface area $\left(\sim 3000 \mathrm{~m}^{2} / \mathrm{g}\right)$, easily accessible hierarchical porosity, high conductivity, and mechanical stability makes activated graphene a very attractive candidate for supercapacitor applications. ${ }^{11-14}$ Gravimetric capacitance values in the range of $90-170 \mathrm{~F} / \mathrm{g}$ have been reported for supercapacitors prepared using a-rGO, ${ }^{11-13}$ with a single report of even higher values of $\leq 326 \mathrm{~F} / \mathrm{g}$. ${ }^{15}$ Attempts to improve the performance of a-rGO electrodes have been reported by variation of precursors, modification of structure, and pore size distribution of these materials. ${ }^{16-18}$ However, relatively complex procedures have always been reported for preparation of arGO electrodes.

Using aqueous a-rGO dispersions for the preparation of high-surface area electrodes could provide significant advan-

Received: January 27, 2020

Accepted: March 12, 2020

Published: March 12, 2020 
tages for possible applications. The dispersions based on some GRM materials have been reported as a precursor for electrode preparation using, e.g., spray deposition, ink jet printing, or simple brush/blade deposition. ${ }^{19-22}$ However, preparation of aqueous a-rGO dispersions is rather challenging due to the rigid $3 \mathrm{D}$ porous structure of the material composed by interconnected highly defected graphene sheets. These sheets cannot be separated from each other without the loss of the high surface area. Moreover, graphene $e^{23,24}$ and a-rGO are strongly hydrophobic. ${ }^{25}$ The most common approach for preparing stable dispersions of hydrophobic graphene relies on liquid phase exfoliation in organic solvents, which are most often rather toxic and undesirable for industrial applications due to environmental concerns. ${ }^{26}$

An alternative approach based on an aqueous medium and various additives would be preferable. ${ }^{26}$ However, this approach has not yet been demonstrated for the preparation of dispersions based on activated graphene.

Here, we report a simple approach that allows the preparation of stable aqueous dispersions with a tunable concentration using high-surface area activated graphene. The dispersions consist solely of environmentally friendly and nontoxic precursor materials available in bulk amounts and allow precise control of the a-rGO concentration starting from diluted to highly concentrated $(>20 \mathrm{mg} / \mathrm{mL})$. The dispersions are compatible with most of the industrial electrode deposition methods, e.g., spray, brush, or blade deposition. Mechanically flexible electrodes prepared by simple drying of the dispersion maintained a high SSA $\left(>1700 \mathrm{~m}^{2} / \mathrm{g}\right)$ and demonstrated high conductivity $(>800 \mathrm{~S} / \mathrm{m})$ and excellent performance in supercapacitors.

The main purpose of this study was to prepare high-surface area graphene-based electrodes for supercapacitors starting from aqueous and environmentally friendly dispersions. The formulation of a graphene dispersion ideal for the preparation of supercapacitors must combine the following properties. The dispersion must be stable and sufficiently concentrated (a few milligrams per milliliter). The concentration and viscosity must be easily adjustable for the specific deposition method. The dispersion must be compatible with standard industrial deposition methods, provide good adhesion of the electrode material to the substrate after solvent evaporation, ideally not breaking even on flexible current collectors, maintain a high surface area directly after deposition and simple evaporation of the solvent, preferably without additional treatments, and provide good conductivity and high density for the best gravimetric and volumetric performance in supercapacitors.

Both rGO and a-rGO are strongly hydrophobic and do not disperse in water without special additives. First, we tested common industrially applied rheology modifiers, fumed silica, and carboxymethylated cellulose, which indeed provide stable aqueous gel-like dispersions when mixed with rGO. However, the adhesion of films deposited using these dispersions on steel was found to be rather poor. Therefore, adhesionpromoting and binding components were added as part of the formulation. The commonly used binders (fluorinated polymer dispersions and styrene butadiene rubber) are electrically insulating, which makes them less suitable for supercapacitor devices. Therefore, we decided to use carbon nanotubes (CNTs) as an alternative electrically conductive binder. $^{27,28}$ The CNTs are known to exhibit excellent mechanical properties and conductivity but are also hydrophobic. The direct dispersion of strongly hydrophobic CNTs in aqueous medium is not possible. However, recent studies demonstrated that graphene oxide (GO) can be used as an efficient dispersing agent for dispersing $\mathrm{rGO}$ and carbon nanotubes in water. ${ }^{2,30}$ Stable dispersions were prepared using GO-stabilized carbon nanotubes as a binder component, rGO as a filler, and fumed silica as a rheology-modifying and thickening additive. Moreover, a similar formulation was found to be very efficient for the preparation of a-rGO and porous carbon dispersions.

The dispersions are prepared in three simple steps. (1) Carbon nanotubes are dispersed in water using a graphene oxide additive using high-shear mixing by ball milling. (2) Fumed silica is added to stabilize the dispersion and to tune its rheological properties. (3) Reduced graphene oxide or lightly milled a-rGO is added to the fluid mixture and milled again.

The rGO dispersions were applied on a steel substrate and air-dried, resulting in a homogeneous film with an SSA of 240 $\mathrm{m}^{2} / \mathrm{g}$ (see the nitrogen adsorption data in Figure S2c). The decrease in SSA relative to that of precursor $\mathrm{rGO}\left[329 \mathrm{~m}^{2} / \mathrm{g}\right.$ (Figure $\mathrm{S} 1 \mathrm{~b})$ ] demonstrates that $\mathrm{rGO}$ sheets partly restack during the preparation of the dispersion and drying. The effect of CNTs [SSA of $1184 \mathrm{~m}^{2} / \mathrm{g}$ (Figure S2)] in preventing restacking was not observed in our experiments, unlike some previous reports on composite rGO-CNT materials. ${ }^{9,31}$

In contrast to rGO, "activated graphene" (a-rGO) is a material with a rigid porous $3 \mathrm{D}$ structure. It cannot be dispersed to "single sheets" and provides a very high surface area $\left[2580 \mathrm{~m}^{2} / \mathrm{g}\right.$ for the sample used in our tests (Figure S2)] because of the combination of a large pore volume and a small pore size (see the Supporting Information for the characterization of precursor a-rGO). ${ }^{25}$ Therefore, the real challenge in the preparation of aqueous dispersions is to make relatively large (micrometer size) grains of a-rGO soluble without breaking their porous structure. Here, we demonstrate that stable aqueous dispersions can be produced using a-rGO filler by using the same procedure that was used for rGO with an only slight adjustment of the relative amount of components.

The design of dispersion composition and the functions of each component are summarized in Figure 1. It provides a

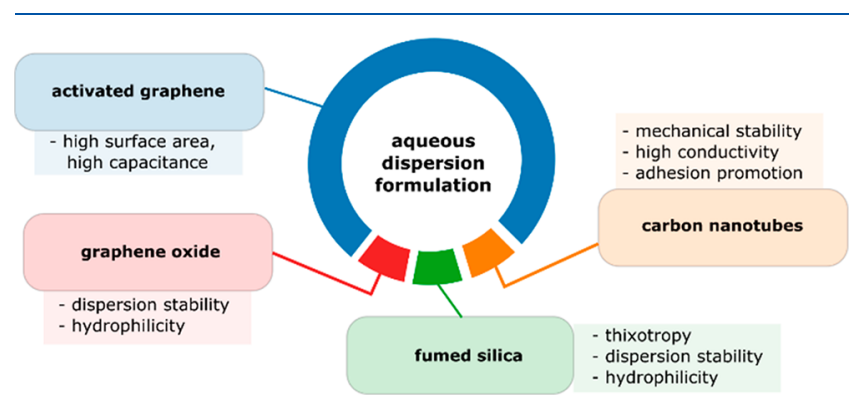

Figure 1. Schematic representation of the aqueous rGO and a-rGO dispersion design for supercapacitor electrodes. The functions of the individual components are noted. The pie chart areas represent the weight content of each component in the dispersion.

rather general approach for the preparation of dispersions/ inks with a broad range of concentrations, density, viscosity, adhesion, conductivity of dried electrodes, and film stability under conditions of flexibility.

The dispersion is based on the balance of four components. The major part is high-surface area graphene-related material (filler). CNTs provide mechanical strength, electrical con- 
ductivity, and connection between a material's grains, while graphene oxide and fumed silica help to stabilize hydrophobic components in an aqueous dispersion. GO is easily adsorbed on the surface of CNTs because of the coexistence of randomly distributed hydrophilic and hydrophobic areas within GO sheets. ${ }^{32,33} \mathrm{CNTs}$ are essentially graphene rolled into a tube. Therefore, a similar mechanism of GO interaction is expected for CNTs, graphene, and a-rGO. The $\pi$-stacking interaction of hydrophobic parts of GO sheets with an activated graphene surface on one side and strong interaction of hydrophilic parts of GO with water on the other side help to make a-rGO particles stable in dispersions. GO is also quite flexible and capable of wrapping grains of an irregular shape. We believe that the ability of GO to wrap and/or envelop both hydrophobic (a-rGO and CNTs) and hydrophilic particles $\left(\mathrm{SiO}_{2}\right)$ helps to improve the stability of dispersions. It is clear that the dispersion properties can be tuned in a rather broad range, providing dispersions for many applications. Using fumed silica and graphene oxide is crucial for the preparation of stable dispersions, but both of these components are not conductive. However, it is well-known that electrically insulating GO can easily be converted into more conductive rGO using a relatively mild heat treatment. $^{34,35}$

The formulations described below were mostly sought for the preparation of supercapacitor electrodes and simple brush/blade deposition. The components shown in Figure 1 were mixed in different proportions and tested for adhesion, conductivity, and surface area. In particular, good results were obtained using a component weight ratio of 10:1:1:1 (arGO:GO: $\mathrm{SiO}_{2}: \mathrm{CNT}$ ).

Both rGO and a-rGO demonstrated the good stability of the fluid dispersion. No visible precipitation was observed even after shelf storage for several days (see Figure $2 \mathrm{~d}$ and the Supporting Information).

The aqueous dispersions (Figure 2a) were deposited onto a flexible stainless steel substrate and vacuum-dried, producing electrodes with typical thicknesses of $\sim 100-500 \mu \mathrm{m}$ (Figure
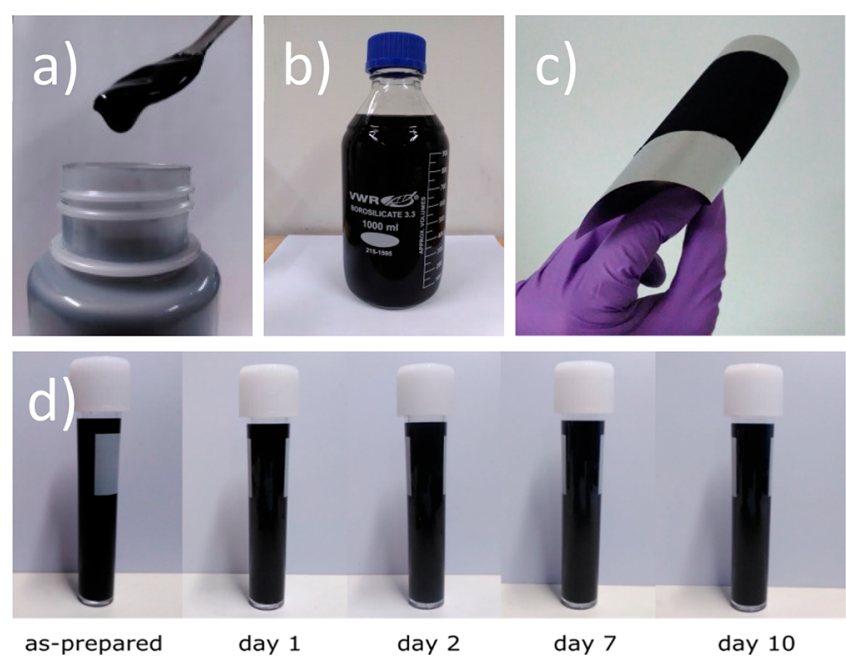

Figure 2. (a) Image showing a dense a-rGO aqueous dispersion. (b) The dispersion can be easily prepared in liter amounts. (c) The arGO dispersion deposited and dried on a flexible stainless steel substrate shows good adhesion even under bending conditions. (d) Dispersions are stable after days of shelf storage showing no significant precipitation.
$3 c)$. The electrodes show good adhesion to the substrate and do not split even after severe bending of steel foil (Figure 2c).

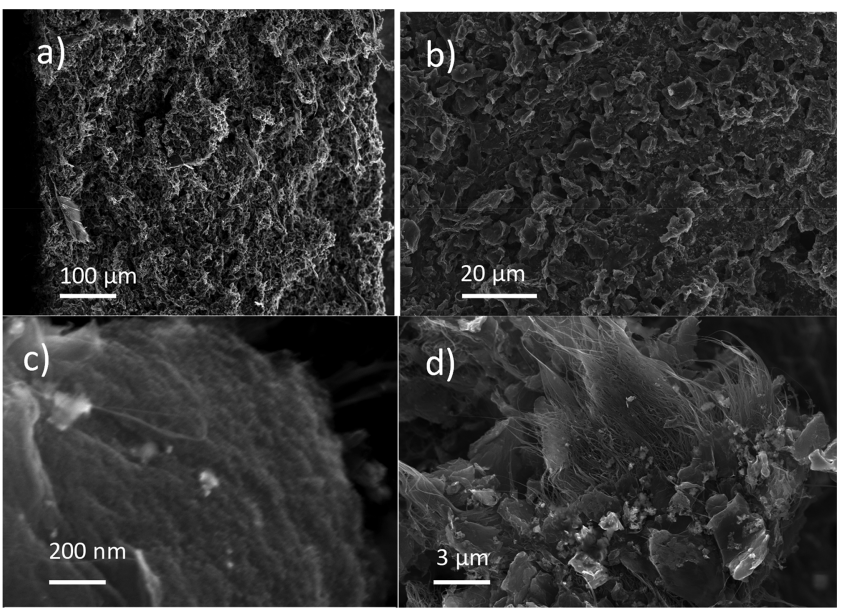

Figure 3. SEM images of dried and annealed at $200{ }^{\circ} \mathrm{C}$ on an a-rGO electrode: (a) cross section, (b) surface, (c) high-magnification image of an a-rGO particle with a porous structure, and (d) micrometersized a-rGO particles interconnected by CNT bundles.

Scanning electron microscopy examination of the high-surface area coating revealed the presence of micrometer-scale particles of activated graphene connected by carbon nanotubes (Figure 3).

A sample of the electrode was peeled off from the steel substrate and used for nitrogen sorption tests. The a-rGObased electrode deposited using an aqueous dispersion exhibited a high BET surface area of $\sim 1720 \mathrm{~m}^{2} / \mathrm{g}$ and a cumulative SSA by QSDFT of $\sim 1050 \mathrm{~m}^{2} / \mathrm{g}$ (Figure 4 ).

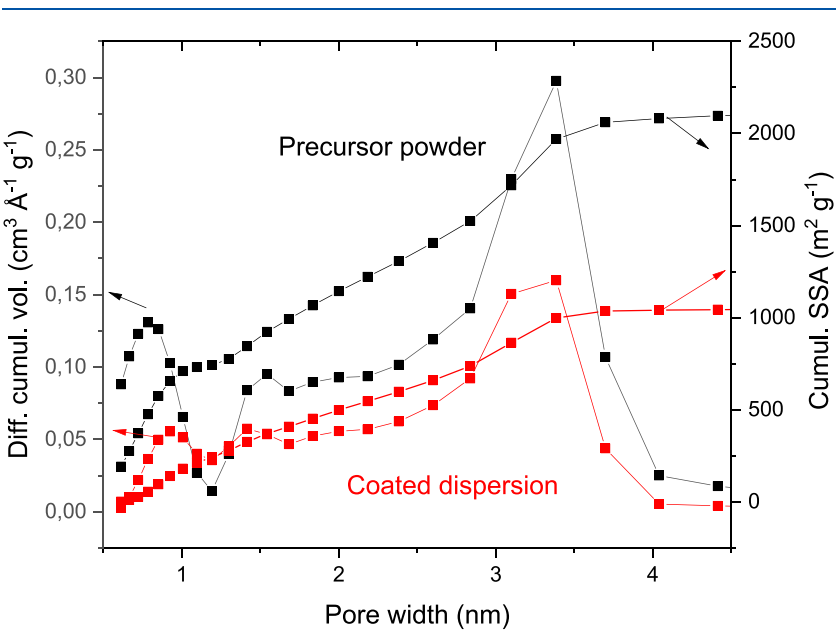

Figure 4. Pore size distribution evaluated using analysis of nitrogen sorption isotherms (QSDFT model) for precursor powder a-rGO and an electrode sample prepared by simple drying of an a-rGO dispersion. The cumulative SSA plot shows that the surface area originates in both samples from $<4 \mathrm{~nm}$ pores.

Remarkably, the coated dispersion showed a pore size distribution very similar to that of precursor powder a-rGO with a pore size of $<4 \mathrm{~nm}$ and a relatively large pore volume $\left(\sim 1 \mathrm{~cm}^{3} / \mathrm{g}\right)$ (Figure 3 ).

One can conclude that the $3 \mathrm{D}$ structure a-rGO survives all treatments involved in the preparation and deposition of the 

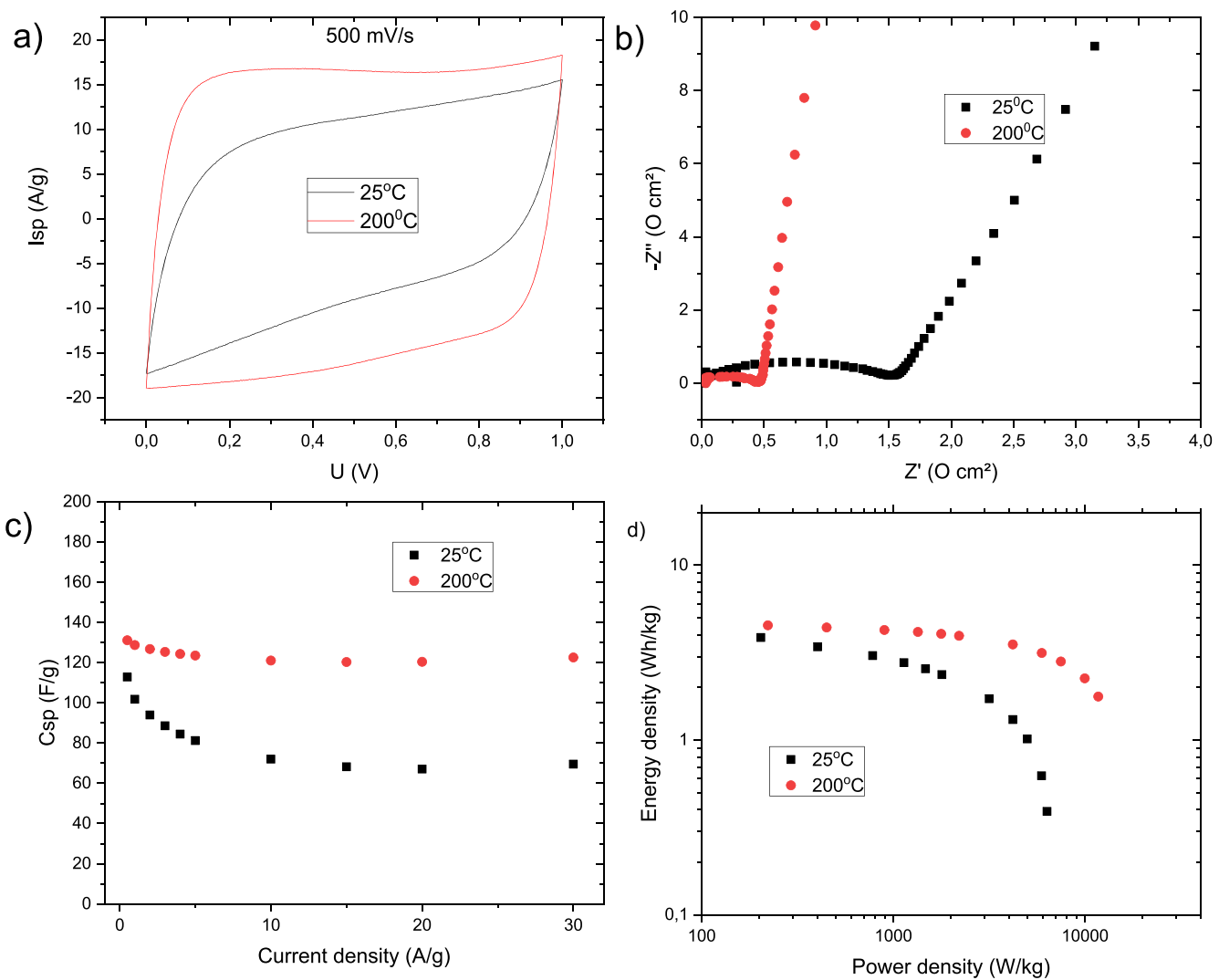

Figure 5. Effect of a-rGO-based electrode annealing on the performance in supercapacitors in a $\mathrm{KOH}$ electrolyte: (a) more square shape of CV curves, (b) better conductivity that is evident from the Nyquist plot (smaller semicircle and a steeper slope of the linear part), (c) improved gravimetric capacitance (especially at high current rates), and (d) increased power and energy density.

dispersion. The electrical conductivity of a-rGO electrodes can be improved by postdeposition annealing at $200{ }^{\circ} \mathrm{C}$, which is above the main step in thermal deoxygenation of precursor GO as demonstrated by thermogravimetric analysis (see Figure S8). A further increase of the annealing temperature to $250{ }^{\circ} \mathrm{C}$ does not provide significant further improvement of electrodes. The rGO flakes formed as a result of the thermal treatment provide better electrical contact between a-rGO grains and significantly improve the overall conductivity of electrodes.

The performance of a-rGO electrodes prepared by coating and drying aqueous dispersions (with and without postdeposition annealing) was evaluated using a two-electrode supercapacitor setup in an aqueous electrolyte (6 M KOH) and an organic electrolyte ( $1 \mathrm{MTEA} \mathrm{BF}_{4}$ in acetonitrile). Relatively high values of specific current and $\mathrm{CV}$ curve shapes close to rectangular were observed directly after coating using not annealed a-rGO electrodes at scanning rates of $\leq 100 \mathrm{mV} /$ $s$, with deviations from the rectangular shape at higher scanning rates (Figure 5 and Figure S12). Almost linear charge-discharge curves confirm that a-rGO-based electrodes demonstrate the predominantly electric double-layer capacitance nature of charge storage (Figure S12). The performance of a-rGO-based electrodes was found to be significantly better compared to that of electrodes prepared using rGO base dispersions already before postdeposition annealing (Figure S15). The high surface area of coated a-rGO electrodes (BET SSA $=1720 \mathrm{~m}^{2} / \mathrm{g}$ ) provides a clear advantage for the performance of supercapacitor electrodes compared to reference coating based on rGO (BET SSA $=240 \mathrm{~m}^{2} / \mathrm{g}$ ).
As a result, the rGO-based electrode coating showed a significantly lower gravimetric capacitance in aqueous and especially in organic electrolytes (Figure S15). More importantly, electrodes based on the low-surface area rGO filler were mechanically unstable during electrochemical characterization and decomposed when tested at high current densities (Figure S15).

As expected, thermal annealing of coated electrodes at 200 ${ }^{\circ} \mathrm{C}$ significantly improved all of the parameters in the performance of a-rGO-based supercapacitors and resulted in a more square shape of $\mathrm{CV}$, a lower ESR, improved operation at high current densities, and an increase in gravimetric capacitance (Figure 5).

The best samples exhibited high specific capacitance values of $\sim 180 \mathrm{~F} / \mathrm{g}(6 \mathrm{M} \mathrm{KOH}$ electrolyte) and $\sim 140 \mathrm{~F} / \mathrm{g}(1 \mathrm{M}$ TEA-BF $F_{4}$ in $\mathrm{AN}$ ) obtained for the annealed a-rGO electrodes (Figure 6).

On the basis of the galvanostatic charge-discharge data, the a-rGO electrodes showed an energy density of $35.6 \mathrm{Wh} / \mathrm{kg}$ and a power density of $42.2 \mathrm{~kW} / \mathrm{kg}$ in a TEA $\mathrm{BF}_{4} / \mathrm{AN}$ electrolyte $(\leq 4.5 \mathrm{Wh} / \mathrm{kg}$ and $\leq 11.7 \mathrm{~kW} / \mathrm{kg}$, respectively, for a $\mathrm{KOH}$ electrolyte) (Figure 6f).

That is comparable to the electrodes prepared earlier using pure a-rGO and relatively complex preparation procedures. ${ }^{11,36}$ Note that the specific energy and specific power values that we report were calculated per unit mass of the electrode, without taking into account the mass of the electrolyte and packaging. The electrolyte and packaging constitute a significant fraction of the total weight of a 

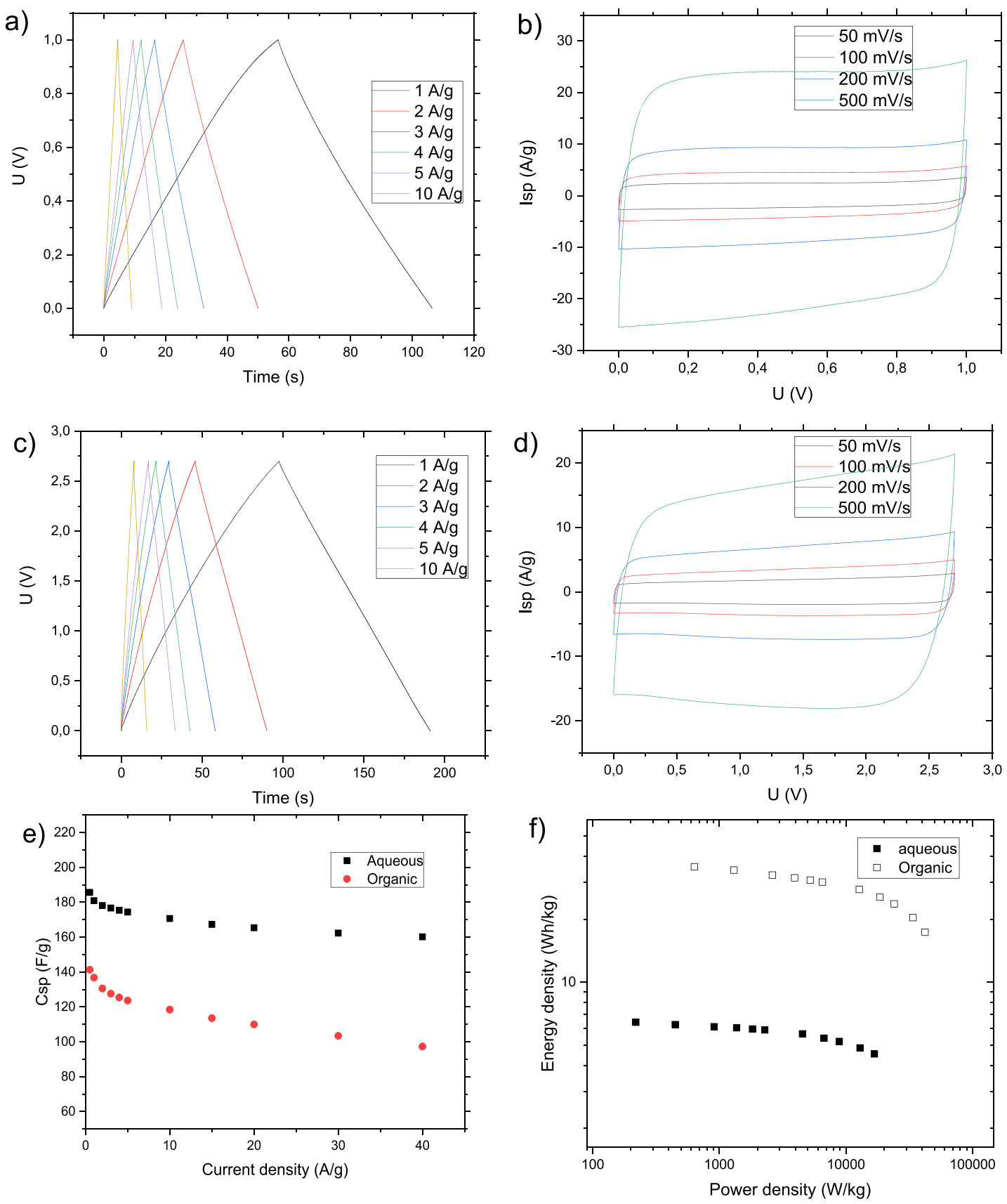

Figure 6. Electrochemical performance of the coated a-rGO electrodes annealed at $200{ }^{\circ} \mathrm{C}$ and measured in a two-electrode configuration using $(\mathrm{a}$ and $\mathrm{b})$ aqueous $(6 \mathrm{M} \mathrm{KOH})$ and $(\mathrm{c}$ and $\mathrm{d}$ ) organic (TEA BF $/ \mathrm{AN}$ ) electrolytes. (b and d) CV curves at varied scanning rates. (a and $c)$ Galvanostatic charge-discharge curves at varied current density values. (e) Specific capacitance vs current density calculated using the galvanostatic discharge data. (f) Ragone plot.

supercapacitor; ${ }^{37}$ therefore, the actual energy storage parameters of a packaged supercapacitor cell will be lower.

It should be noted that heat treatment at $200-250{ }^{\circ} \mathrm{C}$ can easily be introduced directly into the procedure of electrode preparation, e.g., by direct application of dispersion on a hot substrate. The dispersions prepared using the proposed formulations are compatible with various possible deposition methods, including, e.g., spray deposition or ink jet printing. However, the formulations need to be tested and optimized for specific deposition methods, which requires additional studies.

In summary, our study provides the first example of stable aqueous a-rGO dispersions that preserve the main properties of the precursor material (high surface area and good conductivity) after simple coating and drying. The dispersions are compatible with the main industrial methods of electrode deposition such as brush/blade deposition, spray coating, and ink jet printing. Moreover, the electrodes prepared using the coated dispersions provide gravimetric and volumetric capacitance values similar to powder-based a-rGO electrodes $(\sim 180 \mathrm{~F} / \mathrm{g}) .^{12,13,19}$ We suggest that the superior performance of a-rGO electrodes in a supercapacitor is related to a combination of the high surface area of filler material and improved contact between material grains provided by carbon nanotubes. Carbon nanotubes are also easy to bend, which provides the improved flexibility of electrodes (Figure 2c). 
Micrometer-sized a-rGO grains provide the main contribution to the high surface area of electrodes, while CNTs interconnect the grains into flexible conductive electrode material.

\section{ASSOCIATED CONTENT}

\section{(s) Supporting Information}

The Supporting Information is available free of charge at https://pubs.acs.org/doi/10.1021/acs.jpclett.0c00272.

Experimental details and supplementary data (PDF)

\section{AUTHOR INFORMATION}

\section{Corresponding Author}

Alexandr Talyzin - Department of Physics, Umeå University, Umeå SE-901 87, Sweden; 이이.org/0000-0002-33208487; Email: alexandr.talyzin@umu.se

\section{Authors}

Vasyl Skrypnychuk - Department of Physics, Umeå University, Umeå SE-901 87, Sweden

Nicolas Boulanger - Department of Physics, Umeå University, Umeå SE-901 87, Sweden

Andreas Nordenström - Department of Physics, Umeå University, Umeå SE-901 87, Sweden

Complete contact information is available at:

https://pubs.acs.org/10.1021/acs.jpclett.0c00272

\section{Notes}

The authors declare no competing financial interest.

\section{ACKNOWLEDGMENTS}

The authors acknowledge funding from the European Union's Horizon 2020 research and innovation program under grant agreement No785219 and Swedish Research Council Grant 2017-04173. The authors also acknowledge support from the Vibrational Spectroscopy Platform of Umeå University and A. Shchukarev for support with the XPS test. The authors thank Jiantong Li for help with rheology tests.

\section{REFERENCES}

(1) Gonzalez, A.; Goikolea, E.; Barrena, J. A.; Mysyk, R. Review on Supercapacitors: Technologies and Materials. Renewable Sustainable Energy Rev. 2016, 58, 1189-1206.

(2) Yan, J.; Wang, Q.; Wei, T.; Fan, Z. J. Recent Advances in Design and Fabrication of Electrochemical Supercapacitors with High Energy Densities. Adv. Energy Mater. 2014, 4, 1300816.

(3) Wu, Z. S.; Parvez, K.; Winter, A.; Vieker, H.; Liu, X.; Han, S.; Turchanin, A.; Feng, X.; Mullen, K. Layer-by-Layer Assembled Heteroatom-Doped Graphene Films with Ultrahigh Volumetric Capacitance and Rate Capability for Micro-Supercapacitors. Adv. Mater. 2014, 26, 4552-8.

(4) Gilshteyn, E. P.; Kallio, T.; Kanninen, P.; Fedorovskaya, E. O.; Anisimov, A. S.; Nasibulin, A. G. Stretchable and Transparent Supercapacitors Based on Aerosol Synthesized Single-Walled Carbon Nanotube Films. RSC Adv. 2016, 6, 93915-93921.

(5) Shao, Y. L.; El-Kady, M. F.; Wang, L. J.; Zhang, Q. H.; Li, Y. G.; Wang, H. Z.; Mousavi, M. F.; Kaner, R. B. Graphene-Based Materials for Flexible Supercapacitors. Chem. Soc. Rev. 2015, 44, 3639-3665.

(6) Bonaccorso, F.; Colombo, L.; Yu, G. H.; Stoller, M.; Tozzini, V.; Ferrari, A. C.; Ruoff, R. S.; Pellegrini, V. Graphene, Related TwoDimensional Crystals, and Hybrid Systems for Energy Conversion and Storage. Science 2015, 347, 1246501.
(7) Raccichini, R.; Varzi, A.; Passerini, S.; Scrosati, B. The Role of Graphene for Electrochemical Energy Storage. Nat. Mater. 2015, 14, 271-279.

(8) Sun, Y. Q.; Wu, Q. O.; Shi, G. Q. Graphene Based New Energy Materials. Energy Environ. Sci. 2011, 4, 1113-1132.

(9) Wang, Y.; Wu, Y. P.; Huang, Y.; Zhang, F.; Yang, X.; Ma, Y. F.; Chen, Y. S. Preventing Graphene Sheets from Restacking for HighCapacitance Performance. J. Phys. Chem. C 2011, 115, 23192-23197.

(10) Li, J. T.; Ostling, M. Prevention of Graphene Restacking for Performance Boost of Supercapacitors-a Review. Crystals 2013, 3, 163-190.

(11) Kim, T.; Jung, G.; Yoo, S.; Suh, K. S.; Ruoff, R. S. Activated Graphene-Based Carbons as Supercapacitor Electrodes with Macroand Mesopores. ACS Nano 2013, 7, 6899-6905.

(12) Murali, S.; Potts, J. R.; Stoller, S.; Park, J.; Stoller, M. D.; Zhang, L. L.; Zhu, Y. W.; Ruoff, R. S. Preparation of Activated Graphene and Effect of Activation Parameters on Electrochemical Capacitance. Carbon 2012, 50, 3482-3485.

(13) Zhu, Y. W.; et al. Carbon-Based Supercapacitors Produced by Activation of Graphene. Science 2011, 332, 1537-1541.

(14) Klechikov, A.; Mercier, G.; Sharifi, T.; Baburin, I. A.; Seifert, G.; Talyzin, A. V. Hydrogen Storage in High Surface Area Graphene Scaffolds. Chem. Commun. 2015, 51, 15280-15283.

(15) Yin, C. M.; Tao, C. A.; Cai, F. L.; Song, C. C.; Gong, H.; Wang, J. F. Effects of Activation Temperature on the Deoxygenation, Specific Surface Area and Supercapacitor Performance of Graphene. Carbon 2016, 109, 558-565.

(16) Murali, S.; Quarles, N.; Zhang, L. L.; Potts, J. R.; Tan, Z. Q.; Lu, Y. L.; Zhu, Y. W.; Ruoff, R. S. Volumetric Capacitance of Compressed Activated Microwave-Expanded Graphite Oxide (aMego) Electrodes. Nano Energy 2013, 2, 764-768.

(17) Zheng, C.; Zhou, X. F.; Cao, H. L.; Wang, G. H.; Liu, Z. P. Controllable Synthesis of Activated Graphene and Its Application in Supercapacitors. J. Mater. Chem. A 2015, 3, 9543-9549.

(18) Wu, Z. S.; Yang, S.; Zhang, L. L.; Wagner, J. B.; Feng, X. L.; Mullen, K. Binder-Free Activated Graphene Compact Films for AllSolid-State Micro-Supercapacitors with High Areal and Volumetric Capacitances. Energy Storage Mater. 2015, 1, 119-126.

(19) Zheng, H. Q.; Neo, C. Y.; Mei, X. G.; Qiu, J.; Ouyang, J. Y. Reduced Graphene Oxide Films Fabricated by Gel Coating and Their Application as Platinum-Free Counter Electrodes of Highly Efficient Iodide/Triiodide Dye-Sensitized Solar Cells. J. Mater. Chem. 2012, 22, 14465-14474.

(20) Li, J. T.; Ye, F.; Vaziri, S.; Muhammed, M.; Lemme, M. C.; Ostling, M. Efficient Inkjet Printing of Graphene. Adv. Mater. 2013, $25,3985-3992$.

(21) Carey, T.; Jones, C.; Le Moal, F.; Deganello, D.; Torrisi, F. Spray-Coating Thin Films on Three-Dimensional Surfaces for a Semitransparent Capacitive-Touch Device. ACS Appl. Mater. Interfaces 2018, 10, 19948-19956.

(22) Pu, N. W.; Wang, C. A.; Liu, Y. M.; Sung, Y.; Wang, D. S.; Ger, M. D. Dispersion of Graphene in Aqueous Solutions with Different Types of Surfactants and the Production of Graphene Films by Spray or Drop Coating. J. Taiwan Inst. Chem. Eng. 2012, 43, 140146.

(23) Feng, J.; Guo, Z. G. Wettability of Graphene: From Influencing Factors and Reversible Conversions to Potential Applications. Nanoscale Horiz 2019, 4, 339-364.

(24) Taherian, F.; Marcon, V.; van der Vegt, N. F. A.; Leroy, F. What Is the Contact Angle of Water on Graphene? Langmuir 2013, 29, 1457-1465.

(25) Iakunkov, A.; Skrypnychuk, V.; Nordenstrom, A.; Shilayeva, E. A.; Korobov, M.; Prodana, M.; Enachescu, M.; Larsson, S. H.; Talyzin, A. V. Activated Graphene as a Material for Supercapacitor Electrodes: Effects of Surface Area, Pore Size Distribution and Hydrophilicity. Phys. Chem. Chem. Phys. 2019, 21, 17901-17912.

(26) Xu, Y. Y.; Cao, H. Z.; Xue, Y. Q.; Li, B.; Cai, W. H. LiquidPhase Exfoliation of Graphene: An Overview on Exfoliation Media, Techniques, and Challenges. Nanomaterials 2018, 8, 942. 
(27) Xu, G. H.; Zheng, C.; Zhang, Q.; Huang, J. Q.; Zhao, M. Q.; Nie, J. Q.; Wang, X. H.; Wei, F. Binder-Free Activated Carbon/ Carbon Nanotube Paper Electrodes for Use in Supercapacitors. Nano Res. 2011, 4, 870-881.

(28) Perera, S. D.; Patel, B.; Nijem, N.; Roodenko, K.; Seitz, O.; Ferraris, J. P.; Chabal, Y. J.; Balkus, K. J. Vanadium Oxide NanowireCarbon Nanotube Binder-Free Flexible Electrodes for Supercapacitors. Adv. Energy Mater. 2011, 1, 936-945.

(29) Yu, D. S.; Dai, L. M. Self-Assembled Graphene/Carbon Nanotube Hybrid Films for Supercapacitors. J. Phys. Chem. Lett. 2010, 1, 467-470.

(30) Guo, S. R.; Wang, W.; Ozkan, C. S.; Ozkan, M. Assembled Graphene Oxide and Single-Walled Carbon Nanotube Ink for Stable Supercapacitors. J. Mater. Res. 2013, 28, 918-926.

(31) Ansaldo, A.; Bondavalli, P.; Bellani, S.; Del Rio Castillo, A. E.; Prato, M.; Pellegrini, V.; Pognon, G.; Bonaccorso, F. High-Power Graphene-Carbon Nanotube Hybrid Supercapacitors. ChemNanoMat 2017, 3, 436-446.

(32) Zhang, C.; Ren, L. L.; Wang, X. Y.; Liu, T. X. Graphene Oxide-Assisted Dispersion of Pristine Multiwalled Carbon Nanotubes in Aqueous Media. J. Phys. Chem. C 2010, 114, 11435-11440.

(33) Qiu, L.; Yang, X. W.; Gou, X. L.; Yang, W. R.; Ma, Z. F.; Wallace, G. G.; Li, D. Dispersing Carbon Nanotubes with Graphene Oxide in Water and Synergistic Effects between Graphene Derivatives. Chem. - Eur. J. 2010, 16, 10653-10658.

(34) Hofmann, U.; Frenzel, A.; Csalán, E. Die Konstitution Der Graphitsäure Und Ihre Reaktionen. Justus Liebigs Ann. Chem. 1934, $510,1-41$.

(35) Wei, Z. Q.; et al. Nanoscale Tunable Reduction of Graphene Oxide for Graphene Electronics. Science 2010, 328, 1373-1376.

(36) Zhang, L. L.; Zhao, X.; Stoller, M. D.; Zhu, Y. W.; Ji, H. X.; Murali, S.; Wu, Y. P.; Perales, S.; Clevenger, B.; Ruoff, R. S. Highly Conductive and Porous Activated Reduced Graphene Oxide Films for High-Power Supercapacitors. Nano Lett. 2012, 12, 1806-1812.

(37) Gogotsi, Y.; Simon, P. True Performance Metrics in Electrochemical Energy Storage. Science 2011, 334, 917-918. 